\title{
The protein encoded by cancer/testis gene D40/AF15q14 is localized in spermatocytes, acrosomes of spermatids and ejaculated spermatozoa
}

\author{
Takumi Sasao ${ }^{1,2}$, Naoki Itoh ${ }^{2}$, Hiroko Takano ${ }^{3}$, Satoshi Watanabe ${ }^{4}$, Gang Wei ${ }^{1}$, \\ Taiji Tsukamoto ${ }^{2}$, Noboru Kuzumaki ${ }^{1}$ and Masato Takimoto ${ }^{1}$ \\ ${ }^{1}$ Division of Cancer Gene Regulation, Research Section of Disease Control, Institute for Genetic Medicine, \\ Hokkaido University, Kita 15, Nishi 7, Kita-ku, Sapporo, Hokkiaido 060-0815, Japan, ${ }^{2}$ Department of Urology, \\ Sapporo Medical University School of Medicine, Japan, ${ }^{3}$ Department of Functional Morphology, Hokkaido \\ University Graduate School of Medicine, Sapporo, Japan and ${ }^{4}$ Department of Forensic Medicine and Medical \\ Informatics, Hokkaido University Graduate School of Medicine, Sapporo, Japan
}

Correspondence should be addressed to M Takimoto; Email: takimoto@igm.hokudai.ac.jp

G Wei is now at Lankenau Medical Research Center, Wynnewood, Pennsylvania, USA

\begin{abstract}
We have previously identified and cloned a human gene, D40, that is preferentially expressed in testis among normal organs, while it is widely expressed in various human tumor cell lines and primary tumors derived from different organs. In this report, we have examined the expression and localization of this protein in human testis with an antibody specific to D40 protein. In Western analyses, the anti-D40 antibody recognized a major band with a molecular mass of $300 \mathrm{kDa}$ and a minor band of $250 \mathrm{kDa}$. These bands were not observed in the testis lysates from patients with Sertoli-cell-only syndrome and with Kleinfelter syndrome, who lack germ cells of the testis, indicating that D40 protein is expressed in the germ cells of normal testis. Immunohistochemical studies have revealed that D40 protein is highly expressed in spermatocytes and in the pre-acrosome of round spermatids. In the acrosome, D40 protein expression is observed not inside but outside the acrosome membrane. This is consistent with the finding that the amino-acid sequence at the amino terminal of the D40 protein lacks a hydrophobic signal peptide that is required for proteins to translocate to the membrane. Expression of D40 protein is observed in the acrosome of ejaculated spermatozoa as well, although the level is low compared with that in the pre-acrosome of spermatids. These results suggest that D40 protein plays important roles in spermatogenesis, especially in the formation and maintenance of the acrosome.

Reproduction (2004) 128 709-716
\end{abstract}

\section{Introduction}

There is a class of genes which is preferentially expressed in cancer and testis (Boon et al. 1997, Chen \& Old 1999). Some of them were discovered to encode antigens on tumor cells to which immune responses by cytotoxic $\mathrm{T}$ cells or antibodies are elicited in hosts; they are referred to as cancer/testis (CT) antigens. Others, for which the antigenicity remains unknown, are expressed in a similar manner to CT or have homologous sequences to CT. Overall, they may be referred to as CT genes. However, little is known about the physiologic function of these genes in testis. Determining the localization of these proteins in testis could provide important clues to the elucidation of their function.

Previously, we have cloned and reported a human gene, D40, a new cancer/testis gene (Wei et al. 1999, Takimoto et al. 2002). This gene is expressed predominantly in testis among normal organs and tissues, while it is widely expressed in a variety of cultured human cancer cell lines and primary tumors derived from various tissues and organs. Among the primary tumors, D40 expression was observed most frequently in lung cancer. Poorly differentiated lung tumors express D40 more frequently than wellor moderately differentiated ones. In addition, the incidence of D40 expression was significantly higher in tumors from patients who smoke than in those from nonsmokers (Takimoto et al. 2002).

A homology search of the public database indicated that the D40 gene is identical to a gene on human chromosome 15, AF15q14, that is one of the fusion-partner genes of MLL (mixed-lineage leukemia) (Hayette et al. 2000, Chinwalla et al. 2003, Kuefer et al. 2003). MLL gene is often activated by chromosomal translocation in human leukemias (Ayton \& Cleary 2001). The function of 
the D40/AF15q14 gene is elusive, as there is no domain with known function except for a potential nuclear localization signal (Hayette et al. 2000, Takimoto et al. 2002, Kuefer et al. 2003).

D40/AF15q14 cDNA has at least two forms that are probably derived by alternative splicing. One of the cDNAs is about 6 kilo base pairs (kbp) in length and potentially encodes about 1800 amino acids, and the other is longer than $7 \mathrm{kbp}$, encoding more than 2300 amino acids (Hayette et al. 2000, Nagase et al. 2000, Kuefer et al. 2003). They differ in the $3^{\prime}$ regions of the cDNAs and in carboxyl parts of the proteins, but other parts are almost identical except for a short insertion of a sequence in the latter (Nagase et al. 2000, Kuefer et al. 2003).

In this study, we performed experiments to identify D40 protein in human testis with a specific antibody to D40 protein, and then determined the localization of D40 protein in testis. The results show that D40 protein was expressed in the germ cell of testis and that spermatocytes and the acrosome of spermatids express high levels of D40 protein. Interestingly, our data suggest that D40 protein is present outside the acrosome and that the expression of this protein is observed in the acrosome of ejaculated spermatozoa as well. We discuss the potential roles of D40 protein in spermatogenesis and fertilization.

\section{Materials and Methods}

\section{Patients, testis and ejaculated sperm}

Testis samples were obtained from infertile men at times of testicular sperm extraction (TESE) and patients with prostate cancer by castration who underwent surgery at Sapporo Medical University Hospital. The samples were quickly frozen in liquid nitrogen and stored in a deep freezer until protein extracts were prepared. A part of each sample was fixed with Bouin's solution, paraffinembedded and processed for histopathologic examination. The histologic changes of 50 seminiferous tubules in a sample were evaluated. Testicular samples were classified according to clinical and pathologic findings. They included obstructive azoospermia, hypospermatogenesis, maturation arrest, Sertoli-cell-only syndrome (SCO), Klinefelter syndrome, and aged testes from patients with prostate cancer. Ejaculated semen samples for analyses on spermatozoa were obtained from fertile men. All patients gave fully informed consent to the use of their specimens for research. The study was approved by the institutional review board of the university.

\section{Antibody to D40 protein}

A recombinant glutathione S-transferase (GST)-D40 protein, containing amino acids $329-730$ of the D40 protein, was expressed in E. coli with the use of pGEX-3X vector (Amersham-Pharmacia, Aylesbury, UK). The recombinant protein was purified with Glutathione-Sepharose 4B (Amersham-Pharmacia), gel-separated and electroeluted.
The purified protein was injected subcutaneously into a New Zealand White rabbit. Sera were examined for the antibody titer by enzyme-linked immunosorbent assay (ELISA) after the third immunization and then collected after the fourth and fifth immunizations. The binding specificity of the anti-GST-D40 antibody to testicular tissue was confirmed by comparing the reactivities of preimmune rabbit sera, polyclonal anti-GST antibody (AMRAD, Kew, Victoria, Australia), and rabbit immunoglobulin (Ig) (Sigma).

\section{Western blot analysis}

Each testis specimen was lysed in SDS sample buffer (0.5 M Tris, 10\% SDS, 50\% glycerol, 1\% bromophenol blue and 2-mercaptoethanol). Normal testis extract was obtained commercially (Clontech). Fifty micrograms total protein were run on $8 \%$ or $2-15 \%$ gradient protein gel (Daiichi Chemicals, Tokyo, Japan) and blotted onto a nitrocellulose filter (Amersham Pharmacia Biotech, Freiburg, Germany) in a transfer buffer (25 mM Tris, $192 \mathrm{mM}$ glycine and $20 \%(\mathrm{v} / \mathrm{v})$ methanol $(\mathrm{pH} 8.3)$ at $4{ }^{\circ} \mathrm{C}$ for $12 \mathrm{~h}$ at $15 \mathrm{~mA}$. The filter was blocked with Blotto solution (5\% nonfat dry milk, $50 \mathrm{mM}$ Tris-Cl (pH 7.4), $50 \mathrm{mM} \mathrm{NaCl}, 1 \mathrm{mM}$ EDTA and $1 \mathrm{mM}$ DTT) for $1 \mathrm{~h}$ and incubated with the rabbit antiD40 serum (1:5000 in Blotto), and then peroxidase-conjugated goat antirabbit Ig (BIOSOURCE, Camarillo, CA, USA) was used as the second antibody (1:2000 in Blotto), followed by enhanced chemiluminescence (ECL) antibody detection (Amersham). Mouse antihuman $\beta$-actin monoclonal antibody (Chemicon International, Temecula, CA, USA), diluted 1:2000, and peroxidase-conjugated goat antimouse IgG plus IgM $(\mathrm{H}+\mathrm{L})$ (Jackson ImmunoResearch, West Grove, PA, USA), diluted 1:5000, were used to detect $\beta$-actin as an internal control. The detection and quantification of D40 protein was performed with a LAS 1000 (Fuji Film, Tokyo, Japan) and the Image Gauge (Fuji Film) program respectively.

For analyses of ejaculated spermatozoa, sperm samples were washed two times with cold PBS, and the precipitates were lysed in SDS sample buffer. Western blot analyses were performed as above.

\section{Immunohistochemical study}

Tissue sections of testes were deparaffinized, rehydrated and incubated in a boiling antigen-retrieval solution ( $10 \mathrm{mM}$ sodium citrate, $\mathrm{pH} 6.0$ ) for $5 \mathrm{~min}$. Immunohistochemical staining was performed with a Histofine SAB$\mathrm{PO}(\mathrm{R})$ kit (Nichirei, Tokyo, Japan). The sections were incubated for $15 \mathrm{~min}$ in $3 \% \mathrm{H}_{2} \mathrm{O}_{2}$ to kill endogenous peroxidase activity, rinsed in PBS, and incubated in 10\% normal goat serum for $20 \mathrm{~min}$ and then in PBS supplemented with $2.5 \%$ nonfat dry milk for $20 \mathrm{~min}$. The tissue sections were incubated overnight at $4{ }^{\circ} \mathrm{C}$ with either the anti-D40 sera or preimmune rabbit serum at a 1:600 dilution in PBS supplemented with $2.5 \%$ nonfat dry milk. After several washes with PBS, the sections were incubated for $15 \mathrm{~min}$ 
with a biotinylated goat antirabbit $\operatorname{lgG}$, washed again with PBS, and then incubated with peroxidase-conjugated streptoavidin for $5 \mathrm{~min}$. After several rinses with PBS, the sections were incubated with diaminobenzidine (DAB) for $10 \mathrm{~min}$. Nuclei of the sections were counterstained with hematoxylin.

Ejaculated semen was washed three times with mBWW medium by centrifugation, and resuspended and fixed with $0.1 \mathrm{M}$ cacodylate buffer (Wako Pure Chemical, Osaka, Japan) containing $5 \%$ formalin on a slide glass, and then immunohistochemical staining for ejaculated spermatozoa was performed in a manner the same as that with the testis.

\section{Hydropathy analyses}

Hydropathy analyses were performed with DNA/protein sequence analysis software, DNASIS Pro (Hitachi), as described by Kyte and Doolittle (1982).

\section{Results}

For investigation of the D40 protein expression in human testis, Western blot analyses on testis extracts isolated from the patients with infertility were performed with the antibody to D40 protein, as described in Materials and Methods. As patients with obstructive azoospermia have no histologic abnormality in the testis itself, we regard their testes as normal in this study. As castrated testes from patients with prostate cancer are also normal but the patients are usually old, we term such testes 'aged'. The results of the Western blot analyses showed that a major band with a molecular mass of $300 \mathrm{kDa}$ and a minor band of $250 \mathrm{kDa}$ were detected in the testis extracts from the patients with obstructive azoospermia, hypospermatogenesis, maturation arrest and aged testes, and in the normal testis extract that was obtained commercially. D40 protein was detected in all six of the patients with obstructive azoospermia, in three of the six patients with hypospermatogenesis, in two of the four patients with maturation arrest and in 11 of the 14 with aged testes. Representative results of the Western blotting analyses are shown in Fig. 1A. The commercially obtained normal testis extract showed another minor band below the major band.

In contrast, D40 protein was not detected in any of the testis extracts of the 11 patients with SCO syndrome, in the three patients with Kleinfelter syndrome, or in the normal liver and kidney (Fig. 1A). As the patients with these syndromes lack germ cells of the testis, these results indicate that D40 protein is expressed in the germ cells of the testis.

In addition to testis, D40 expression in the male reproductive organs, such as the epididymis and prostate gland, was also examined to reveal the specific expression of D40 protein in the testis. The result showed that D40 protein was detectable in neither the epididymis nor the prostate gland, as shown in Fig. 1B. This result also suggests that D40 protein plays an important role in spermatogenesis in the testis.

To determine the localization of D40 protein expression in normal testis, we performed immunohistochemical studies with the anti-D40 antibody. The testes of the patients with obstructive azoospermia were used in this experiment, as they are histologically normal. The results showed Sertoli cells and Leydig cells to be negative for the D40 staining and spermatogonia to be immunoreactive but stained weakly with the anti-D40 antibody. However, nuclei of primary spermatocytes and acrosomes of spermatids, so-called pre-acrosomes, were intensely stained (Fig. 2A). The D40 protein expression in round spermatids was observed even in the Golgi phase, which is a very early stage of acrosome formation (Fig. 2C) and in a subsequent cap phase (Fig. 2D).

Importantly, enlarged pictures of acrosomes in round spermatids showed that D40 protein expression was not present in the lumen of acrosomes (Fig. 3A). The aminoacid sequence of the amino-terminal D40 protein, deduced from the cDNA, has no signal peptide sequence that consists of hydrophobic amino acids and is required for the protein to translocate to the hydrophobic membrane (Table 1). Hydropathy analyses of the amino-acid sequence of D40 protein were performed, and the results indicated that the amino-acid sequences of both isoforms of D40 protein, which we term D40/AF and D40/K, are hydrophilic overall (Fig. 4). These results suggest that most parts of the D40 protein are located on the cytoplasmic side of the acrosome, although we cannot exclude the possibility that a part of the protein is present inside the acrosomal membrane.

D40 protein expression was examined immunohistochemically in ejaculated spermatozoa derived from normal men. The results showed that D40 protein expression was observed in the acrosomal region of ejaculated spermatozoa (Fig. 5A). This result is consistent with that obtained in Western blotting analyses of the extracts of normal ejaculated spermatozoa showing the presence of D40 protein in such spermatozoa, although the amount is reduced compared with that in testis. The size of D40 protein in spermatozoa is about $220 \mathrm{kDa}$, smaller than that in testis (Fig. 5C).

\section{Discussion}

In this study, we identified D40 protein expression in human testis and ejaculated spermatozoa by biochemical and immunohistochemical methods, using a specific antibody to D40 protein that we developed. The results showed that D40 protein is highly expressed in spermatocytes and pre-acrosomes of spermatids of testis, and in acrosomes of ejaculated spermatozoa.

In Western blot analyses of testis extracts, a major band of $300 \mathrm{kDa}$ and a minor band of $250 \mathrm{kDa}$ were identified. There are two alternative isoforms of D40 cDNA, which we term D40/AF and D40/K, with deduced amino-acid 
A
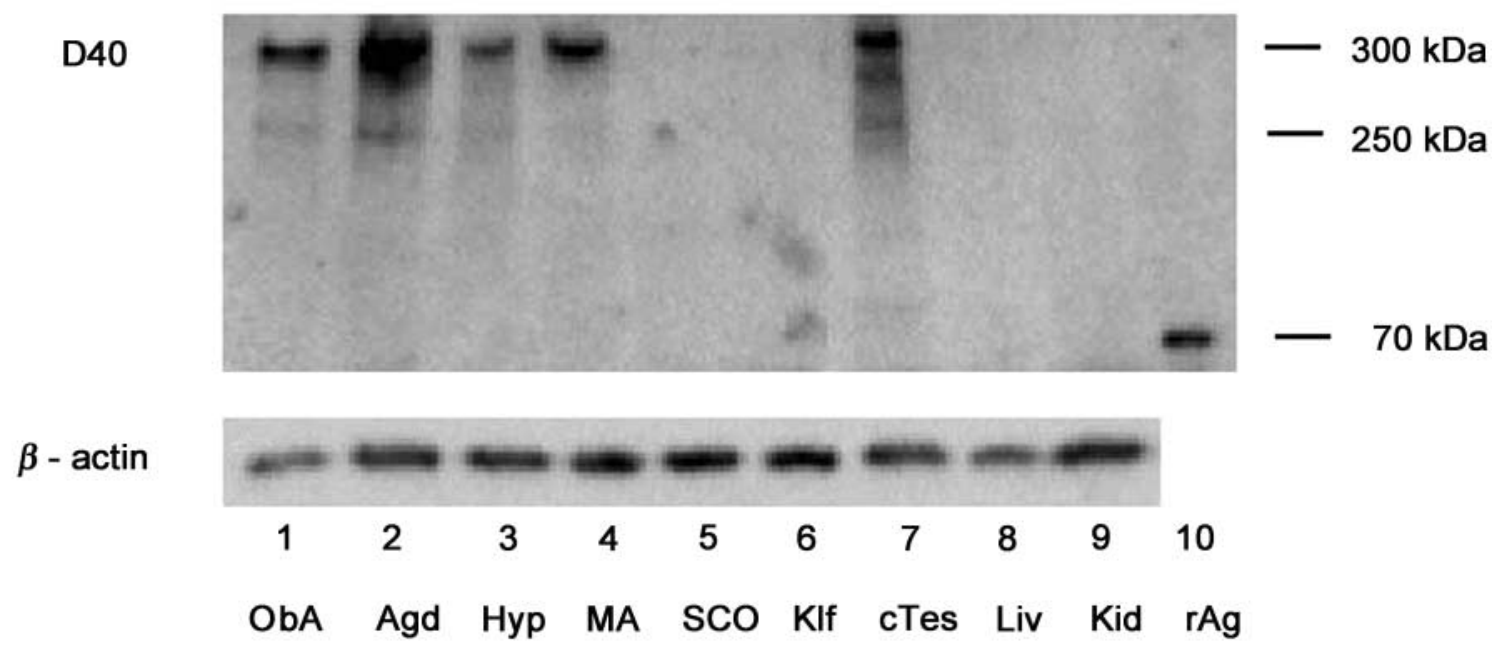

B

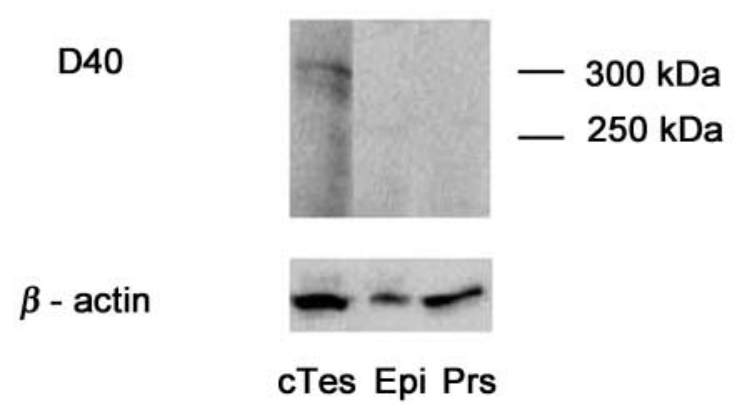

Figure 1 D40 protein expression in human testes. (A) Western blot analysis with anti-D40 antibody was preformed as described in Materials and Methods. Sample testes from lanes 1-6 were derived from patients with obstructive azoospermia (lane 1, ObA), aged testis (lane 2, Agd), hypospermatogenesis (lane 3, Hyp), maturation arrest (lane 4, MA), Sertoli-cell-only syndrome (lane 5, SCO), and Klinefelter syndrome (lane 6, Klf). Control samples were as follows: normal testis obtained commercially (lane 7, cTes) and normal liver (lane 8, Liv) and kidney (lane 9, Kid) as negative controls; recombinant GST-D40 protein used for raising antibody (lane 10, rAg) was the positive control. Numbers on the right side of figure indicate the D40 proteins with molecular masses of 300 and $250 \mathrm{kDa}$ and the GST-D40 protein of $70 \mathrm{kDa}$. Anti- $\beta$-actin antibody was used as a control. (B) D40 protein expression was examined in the epididymis and prostate gland by Western blot analysis, performed as in panel A. Western blot analysis with antiactin antibody was also performed to show the integrity of the extracts. Epi: epididymis; Prs: prostate.

numbers of 1833 and 2316 respectively (Hayette et al. 2000, Kuefer et al. 2003). It is likely that the two bands observed in Western analyses conform to the isoforms of D40 protein, D40/K and D40/AF, assuming the presence of a post-translational modification. The normal testis extract that was obtained commercially showed another minor band below the major one in Western analysis. This may be derived from degradation of the major band, as the extract was probably not prepared immediately after the death of the donor by accident. Importantly, D40 proteins were not detected in the testes of the patients with SOC syndrome and Klinefelter syndrome, who lack germ cells of the testis, indicating that D40 protein is expressed in the germ cell.

For D40 protein in ejaculated spermatozoa, the molecular mass of the detected band is about $220 \mathrm{kDa}$, which is smaller than those detected in testis. This is probably due to a change of post-translational modification of D40 protein, such as potential loss of phosphorylation or protein processing during spermiogenesis.

Immunohistochemical studies also revealed significant D40 expression in germ cells of the testis, especially in spermatocytes and spermatids. Primary spermatocytes showed strong D40 expression, and the D40 protein appears to be present in the nucleus of spermatocytes. This is consistent with the finding that D40 protein has a sequence that is highly homologous to a known nuclear localization signal (Gorlich 1997, Yoneda 1997). As a major part of meiosis occurs in the spermatocyte, it is possible that D40 plays important roles in the process of meiosis as a nuclear protein in spermatocytes.

In spermatids, the so-called pre-acrosomal region of round spermatids was significantly positive for D40 protein expression, even in the very early stage of the 

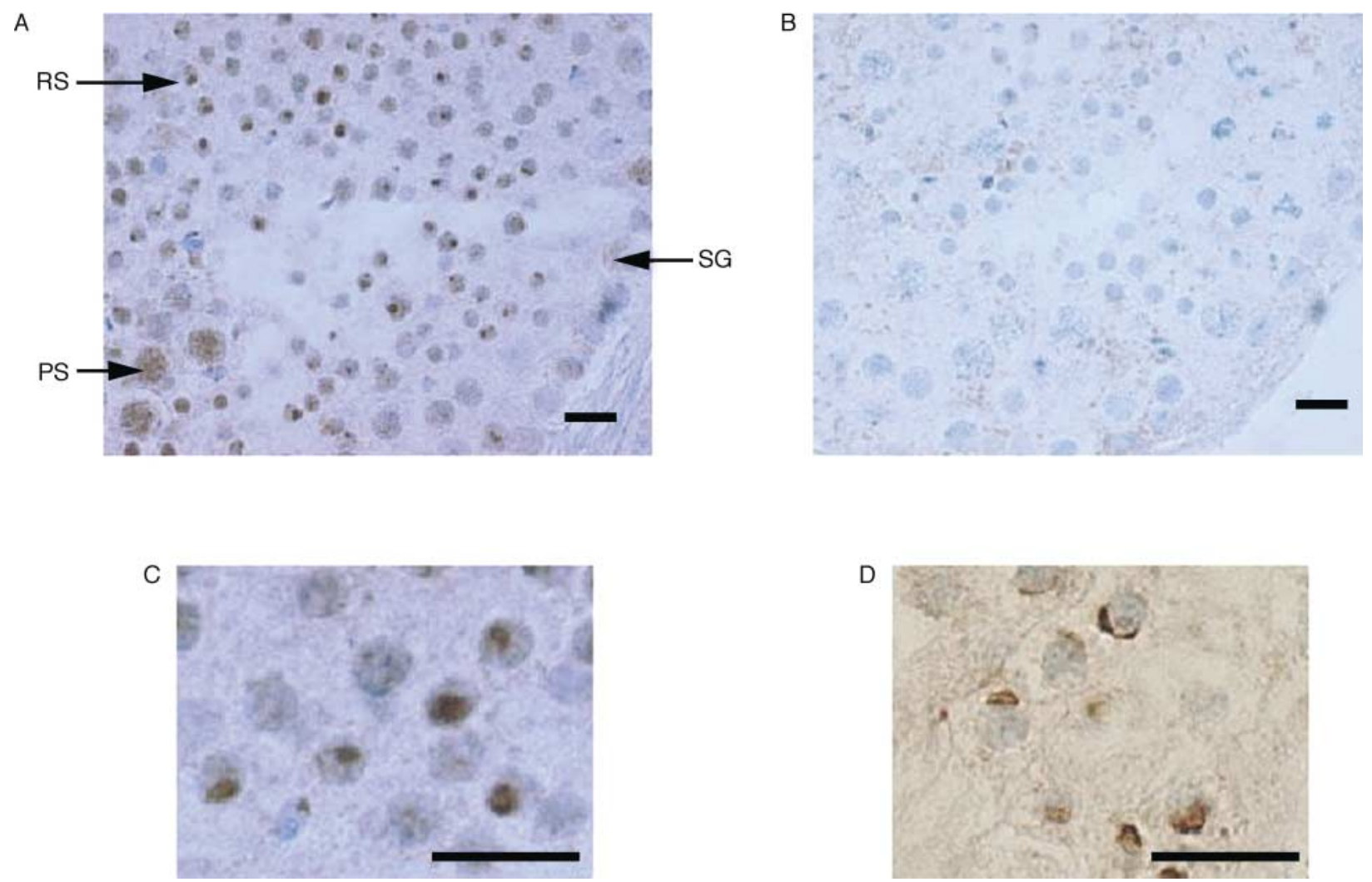

Figure 2 Immunohistochemical staining of D40 protein. Immunohistochemical stainings were performed in the testes of patients with obstructive azoospermia, using the anti-D40 antibody, as described in Materials and Methods. Testicular tissues stained with anti-D40 antisera and with preimmune sera are shown in panels $A$ and $B$ respectively, magnification $=\times 400$. Enlarged images of the Golgi phase and cap phase of the acrosome in round spermatids stained with the anti-D40 antibody are shown in panels C and D respectively, at higher magnification ( $\times 1000)$. SG: spermatogonia; PS: primary spermatocytes; RS: round spermatids. Scale bars $=20 \mu \mathrm{m}$.

acrosome formation, the Golgi phase of round spermatids, and the subsequent cap phase of the spermatids (Bloom \& Fawcett 1994). These results suggest that D40 protein plays an important role in the formation of the acrosome in spermatids.

More than a dozen acrosomal proteins have been identified in mammals. Some of them are enzymes with hydrolytic activity, such as proteinases (Bloom \& Fawcett 1994). Upon fertilization, the acrosome reaction breaks the outer membrane of the acrosome and releases intra-acrosomal proteins that digest the outer surface membrane of the oocyte, the zona pellucida. Some acrosomal proteins, such as SP-10, play a role in binding sperm to the cell membrane of the oocyte (Hamatani et al. 2000).

The intra-acrosomal proteins, such as acrosin, have a hydrophobic amino-acid sequence, signal peptide, at their amino terminals, as shown in Table 1 (Baba et al. 1989). In immunohistochemical studies, D40 protein expression was not observed in the lumen of the acrosomes,
A

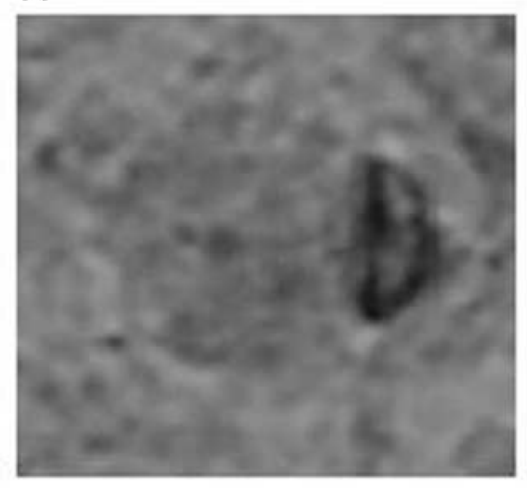

B

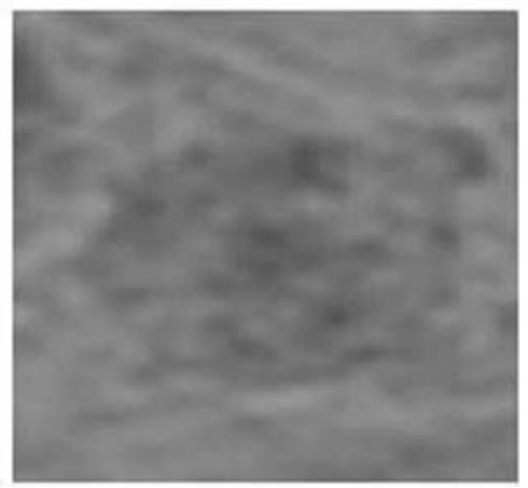

Figure 3 Enlarged image of a cap-phase acrosome in round spermatid. Immunohistochemical staining was performed on the testis of patients with obstructive azoospermia, as in Fig. 2, stained with anti-D40 antisera (A) and with preimmune sera (B). The inside of the lumen of the acrosome in panel $\mathrm{A}$ is not stained with the anti-D40 antibody. Magnification $=\times 1000$. 
Table 1 Amino-terminal amino-acid sequence of acrosomal proteins.

\begin{tabular}{llcc}
\hline Protein & \multicolumn{1}{c}{ Amino-terminal sequence } & Signal peptide & Localization at acrosome \\
\hline Acrosin & MVEMLPTAILLVLAVSVVAKDNATCDGPCG & + & inside \\
SP32 & MRKPAAGFLPSLKVLLLPLAPAAAQDSTQ & + & inside \\
SP10 & MKELILLGLYLLGSSRGAPPGQPDELLDSV & + & inside \\
AZ1 & MKGSRTITATPEGSPEAVDLSLIGLPPPMS & - & outside \\
D40 & MDGVSSEANEENDNIERPVRRRHSSILKPP & - & outside \\
\hline
\end{tabular}

The first 30 amino acids of the proteins are shown. Underlining indicates the signal peptide sequences that are rich in hydrophobic amino acids.

suggesting that D40 protein localizes not inside but outside acrosomes. This observation is consistent with the fact that proteins that are able to translocate to a hydrophobic membrane need a hydrophobic signal peptide at the amino terminus, and that D40 protein, as opposed to intra-acrosomal protein, has no such amino-acid sequence (Table 1) (Blobel 1995, Lodish et al. 2000).

Physiologic roles in the testis of most of the CT genes are not characterized, and there are few reports on the intratesticular localization of CT gene products. Some CT proteins were shown to be present in spermatogonia and primary spermatocytes (Takahashi et al. 1995, Jungbluth et al. 2001). No acrosomal protein was identified as a CT antigen except for the D40 protein revealed in this study and proacrosin-binding protein sp32 precursor (Ono et al. 2001).

One of the intriguing observations in this study is the presence of D40 protein in ejaculated spermatozoa. Only a limited number of proteins are expressed in spermatozoa, as the transcription and protein synthesis decrease and subsequently stop during spermiogenesis. It is reported that expression of AZ-1, an extra-acrosomal protein, like D40, is detectable only in round spermatids (Aoto et al. 1995). Our experimental data obtained in
Western analyses and immunohistochemical studies showed the presence of D40 protein in ejaculated spermatozoa. As the D40 protein is localized outside the acrosome, and the inner membrane of the acrosome does not break with acrosome reaction, it is likely that D40 is still present between the acrosome and nucleus, the so-called perinuclear region of ejaculated spermatozoa, after the reaction. What is the role of D40 protein in ejaculated spermatozoa, especially in the perinucleus?

Several proteins are known to localize to the perinuclear region of spermatozoa. These include SPE-11 in the worm Caenorhabditis elegans, STAT4 in mouse and SubH2Bv in bull (Browning \& Strome 1996, Herrada \& Wolgemuth 1997, Aul \& Oko 2001). Interestingly, they are nuclear proteins, like D40. SPE-11 protein in C. elegans localizes in the nucleus in spermatocytes and perinucleus in sperm. It is reported that sperm of SPE-11 mutant worm are able to fertilize oocytes, but subsequent embryogenesis is abnormal (Browning \& Strome 1996). STAT4 protein is a transcription factor expressed predominantly in the testis of mouse, being localized not only in spermatids but in a perinuclear region of spermatozoa in epididymis (Herrada \& Wolgemuth 1997). SubH2Bv is a histone H2B variant and contains a histone fold motif and bipartite

D40/AF

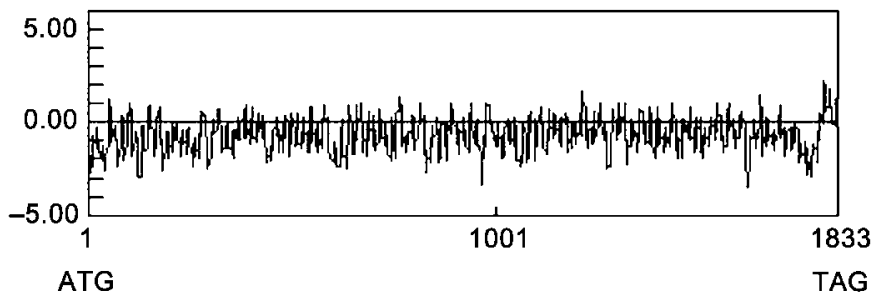

D40/K

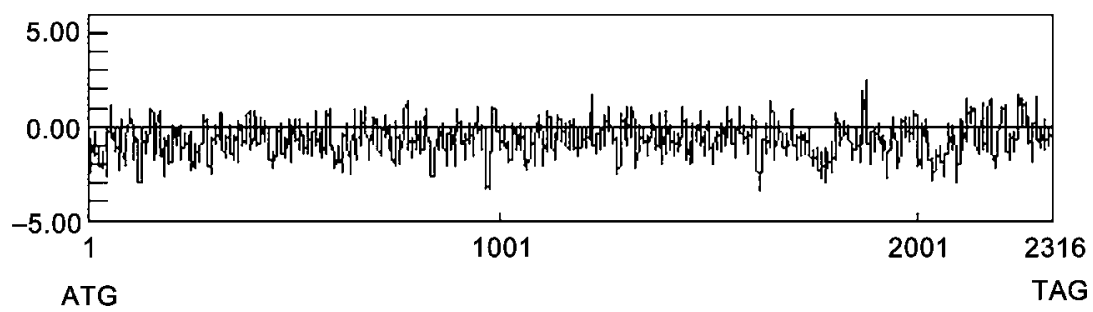

Figure 4 Hydrophobicity plot generated from the deduced amino-acid sequence of D40 protein. The scales and numbers on the ordinate indicate the degree of hydrophobicity of the amino-acid residues in D40 protein. The hydrophobic residues lie above the central line while the hydrophilic residues lie below the line. The numbers under the abscissa are the positions of the amino acids of two isoforms of D40 protein, D40/AF and D40/K. ATG and TAG are the initiation and stop codons respectively. 
A

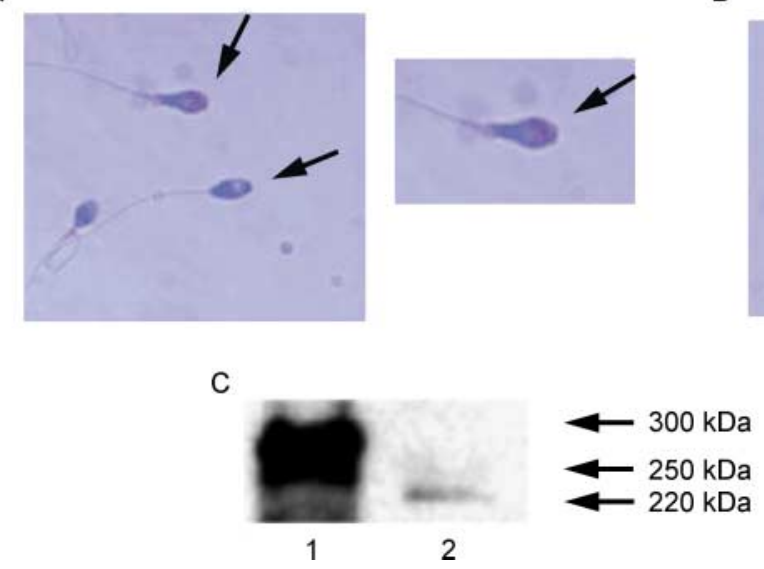

B

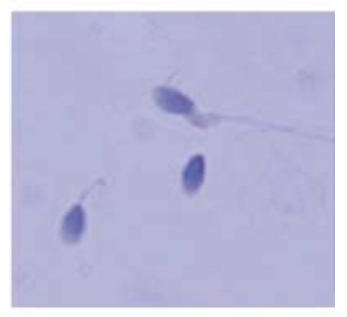

Figure 5 Presence of D40 protein in ejaculated spermatozoa. (A) Ejaculated spermatozoa of fertile men stained with anti-D40 antisera. An enlarged picture of a spermatozoon in which the acrosomal region was stained is shown on the right. Arrows indicate positive staining of acrosomal regions. (B) Negative staining of ejaculated spermatozoa with preimmune sera. (C) Western blot analysis with anti-D40 antibody was performed with lysates from the testis of a patient with obstructive azoospermia (lane 1) and ejaculated spermatozoa of a normal male (lane 2). Arrows with numbers indicate the D40 protein with molecular mass.

nuclear localization signal in its amino-acid sequence (Aul \& Oko 2001). These mammalian proteins are thought to play roles in gene regulation after they are transferred to oocytes upon fertilization.

As D40 protein localizes in different parts of male germ cells, it is a very interesting hypothesis that D40 protein plays important roles in meiosis, fertilization and embryogenesis.

\section{Acknowledgements}

We thank Dr K. Abe, Dr M. Yamashita, Dr K. Mita, Dr T. Baba, the members of the Division of Cancer Gene Regulation, Institute for Genetic Medicine, Hokkaido University, and the members of the Department of Urology, Sapporo Medical University, for valuable discussion. This study was supported by grants-in-aid from the Ministry of Education, Science, Culture, and Sport of Japan, from Ono Cancer Foundation, from Hokkaido Foundation for the Promotion of Scientific and Industrial Technology (Hokscitec), from the Foundation for Promotion of Cancer Research in Japan, from Osaka Cancer Foundation and from the Suhara Memorial Foundation.

\section{References}

Aoto H, Tsuchida J, Nishina $\mathrm{Y}$, Nishimune $\mathrm{Y}$, Asano A \& Tajima S 1995 Isolation of a novel cDNA that encodes a protein localized to the pre-acrosome region of spermatids. European Journal of Biochemistry 234 8-15.

Aul RB \& Oko RJ 2001 The major subacrosomal occupant of bull spermatozoa is a novel histone $\mathrm{H} 2 \mathrm{~B}$ variant associated with the forming spermiogenesis. Developmental Biology 239 376-387.

Ayton PM \& Cleary ML 2001 Molecular mechanisms of leukemogenesis mediated by MLL fusion proteins. Oncogene $\mathbf{2 0}$ 5695-5707.

Baba T, Watanabe K, Kashiwabara S \& Arai Y 1989 Primary structure of human proacrosin deduced from its CDNA sequence. FEBS Letters 244 296-300.
Blobel G 1995 Unidirectional and bidirectional protein traffic across membranes. Cold Spring Harbor Symposia on Quantitative Biology $601-10$.

Bloom W \& Fawcett DW 1994 Male reproductive system. In A Textbook of Histology, 12th edn, pp 768-815. New York: Chapman \& Hall.

Boon T, Coulie PG \& Van den Eynde B 1997 Tumor antigens recognized by T cells. Immunology Today 18 267-268.

Browning H \& Strome S 1996 A sperm-supplied factor required for embryogenesis in C elegans. Development 122 391-404.

Chen Y-T \& Old LJ 1999 Cancer-testis antigens; targets for cancer immunotherapy. Cancer Journal from Scientific American $\mathbf{5}$ $16-17$.

Chinwalla V, Chien A, Odero M, Neilly MB, Zeleznik-Le NJ \& Rowley JD 2003 A $t(11 ; 15)$ fuses MLL to two different genes, AF15q14 and a novel gene MPFYVE on chromosome 15. Oncogene 22 1400-1410.

Gorlich D 1997 Nuclear protein import. Current Opinion in Cell Biology 9 412-419.

Hamatani T, Tanabe K, Kamei K, Sakai N, Yamamoto $\mathrm{Y}$ \& Yoshimura Y 2000 A monoclonal antibody to human SP-10 inhibits in vitro the binding of human sperm to hamster oolemma but not to human zona pellucida. Biology of Reproduction 62 1201-1208.

Hayette S, Tigaud I, Vanier A, Martel S, Corbo L, Charrin C, Beillard E, Deleage G, Magaud JP \& Rimokh R 2000 AF15q14, a novel partner gene fused to the MLL gene in an acute myeloid leukaemia with a $\mathrm{t}(11 ; 15)(\mathrm{q} 23 ; \mathrm{q} 14)$. Oncogene 19 4446-4450.

Herrada G \& Wolgemuth DJ 1997 The mouse transcription factor Stat4 is expressed in haploid male germ cells and is present in the perinuclear theca of spermatozoa. Journal of Cell Science $\mathbf{1 1 0}$ $1543-1553$.

Jungbluth AA, Chen YT, Stockert E, Busan KJ, Kolb D, Iversen K, Coplan K, Williamson B, Altorki N \& Old LJ 2001 Immunohistochemical analysis of NY-EO-1 antigen expression in normal and malignant human tissue. International Journal of Cancer 92 856-860.

Kuefer MU, Chinwalla V, Zeleznik-Le NJ, Behm FG, Naeve CW, Rakestraw KM, Mukatira ST, Raimondi SC \& Morris SW 2003 Characterization of the MLL partner gene AF15q14 involved in $\mathrm{t}(11 ; 15)(\mathrm{q} 23 ; \mathrm{q} 14)$. Oncogene 22 1418-1424. 
Kyte J \& Doolittle RF 1982 A simple method for displaying the hydropathic character of a protein. Journal of Molecular Biology 157 105-132.

Lodish H, Berk A, Zipursky SL, Matsudaira P, Baltimore D \& Darnell J 2000 Protein sorting: organelle biogenesis and protein secretion. In Molecular Cell Biology, 4th edn, pp 675-750. New York: WH Freeman.

Nagase T, Kikuno R, Nakayama M, Hirosawa M \& Ohara O 2000 Prediction of the coding sequences of unidentified human genes. XVIII. The complete sequences of 100 new cDNA clones from brain which code for large proteins in vitro. DNA Research 7 273-281.

Ono T, Kurashige T, Harada N, Noguchi $Y$, Saika T, Niikawa N, Aoe M, Nakamura S, Higashi T, Hiraki A, Wada H, Kumon H, Old LJ \& Nakayama E 2001 Identification of proacrosin binding protein sp32 precursor as a human cancer/testis antigen. PNAS 98 3282-3287.

Takahashi K, Shichijo S, Noguchi M, Hirohata M \& Itoh K 1995 Identification of MAGE-1 and MAGE-4 proteins in spermatogonia and primary spermatocytes of testis. Cancer Research $\mathbf{5 5}$ $3478-3482$.

Takimoto M, Wei G, Dosaka-Akita H, Mao P, Kondo S, Sakuragi N, Chiba N, Miura T, Itoh N, Sasao T, Koya RC, Tsukamoto T, Fujimoto S, Katoh H \& Kuzumaki N 2002 Frequent expression of new cancer/testis gene D40/AF15q14 in lung cancer of smoker. British Journal of Cancer 86 1757-1762.

Wei G, Takimoto M, Yoshida I, Mao P, Koya CR, Miura T \& Kuzumaki N 1999 Chromosomal assignment of a novel gene D40. Nucleic Acid Symposium Series 42 71-72.

Yoneda Y 1997 How proteins are transported from cytoplasm to the nucleus. Journal of Biochemistry 121 811-817.

Received 14 May 2004

First decision 7 July 2004

Revised manuscript received 12 August 2004

Accepted 8 September 2004 